\section{Spontaneous ipsilateral subconjunctival hemorrhage and the related risk factors}

\author{
Evgenia Kanonidou, ${ }^{1}$ Vasileios Konidaris, ${ }^{2}$ \\ Christina Kanonidou, ${ }^{3}$ Nikolas Ziakas ${ }^{2}$ \\ 'Department of Ophthalmology, General \\ Hospital of Veria, Veria; ${ }^{2}$ Department of \\ Ophthalmology, Aristotle University of \\ Thessaloniki, AHEPA University Hospital; \\ ${ }^{3}$ Postgraduate Student, Aristotle \\ University of Thessaloniki, Thessaloniki, \\ Greece
}

\section{Abstract}

The aim of the report is to assess the risk factors among patients with spontaneous ipsilateral subconjunctival hemorrhage (SCH) who presented to the outpatients' department in General Hospital of Veria, Veria, Greece. Thirty-five patients with SCH participated in the study. A thorough case history was taken and a full ophthalmic examination was performed to identify the risk factors related to the clinical finding. The common hematological parameters associated with the coagulation profile of each patient were evaluated. With the exception of $\mathrm{SCH}$, the ophthalmic examination was normal in all patients. Identified risk factors include history of systemic hypertension (21 patients [60\%], mean systolic value: $170 \mathrm{mmHg} \pm 15 \mathrm{mmHg}$ ), strenuous exercise [19 patients (54\%)] and minor ocular trauma [5 patients (14\%)]. Other risk factors [each in 2 patients (6\%)] included: diabetes mellitus, smoking, severe cough, straining at stool, and weight lifting. Seven patients (20\%) were under medication related to bleeding diathesis. The values of the blood coagulation parameters were within the normal limits in all patients. Twenty-nine patients (83\%) had elevated blood pressure during the ophthalmological examination. Our study provides documentation regarding the potential risk factors associated with $\mathrm{SCH}$. It is interesting to observe the high incidence of hypertension among the patients with SCH. Therefore, it is highly recommended that the blood pressure be checked in all patients with SCH and that the patients be referred to a general practitioner for further management if indicated.

\section{Introduction}

Subconjunctival hemorrhage (SCH) is a commonly presenting clinical problem for an ophthalmologist. ${ }^{1,2}$ In several studies in general ophthalmologic studies, SCHs were seen in $0.35-0.8 \%$ of patients. ${ }^{3,4}$ The clinical sign of $\mathrm{SCH}$ is the presence of blood underneath the conjunctiva, often in one sector of the eye, to the extent in some cases that the entire view of the sclera is obstructed. ${ }^{1}$ The purpose of this study was to assess the risk factors among patients with spontaneous ipsilateral SCH who presented to the outpatient department of our referral centre.

\section{Materials and Methods}

Participants in the study were 35 patients (21 male and 14 female), mean age 57 years old $(\mathrm{SD} \pm 16)$, who presented to the ophthalmology outpatient department with spontaneous ipsilateral SCH. A thorough case history was taken from each individual and a full ophthalmic examination (slitlamp and non-contact lens fundus examination) was performed to identify the potential risk factors related to the clinical finding of SCH. The patients' blood pressure was measured at the time of presentation. The common hematological parameters associated with the coagulation profile of each patient [i.e. platelet count (PLT) Prothrobin time (PT), activated partial thromboplastin time (aPTT), and international normalized ratio (INR)] were also evaluated. The study followed the tenets of the Declaration of Helsinki and was approved by the local ethical committee. Written informed consent was obtained from all subjects prior to their participation.

\section{Results}

The ophthalmic examination was normal in all the patients, with the exception of SCH. Twenty-one patients (60\%) had a history of systemic hypertension, 2 (6\%) had a history of diabetes and $2(6 \%)$ were smokers. Nineteen patients (54\%) had a history of recent heavy exercise; 5 patients (14\%), possible minor local trauma during sleep; 2 patients (6\%), severe coughing prior to the subconjunctival bleeding; 2 patients (6\%), straining at stool; and, 2 patients (6\%), lifting of weights. Seven patients (20\%) were under medication related to bleeding diathesis (5 with acetylsalicylic acid and 2 with clopidogrel bisulfate) (Figure 1). In some of the patients there was a co-existence of two or more of the risk factors under investigation. In 19 of the patients there was only one identified risk factor for SCH. In 8 of the patients, there were 2 risk factors (in 5 of the patients, the 2 were hypertension and heavy exercise, in 1 patient, the 2 were hyper-
Correspondence: Kanonidou, Evgenia Department of Ophthalmology, General Hospital of Veria/ 97 Vlasi Gavriilidi Street, GR 55131, Thessaloniki, Greece. Tel: +30.6974416953 .

E-mail: evkanon@hotmail.com

Key words: subconjunctival, hemorrhage, risk factors, hypertension, bleeding diathesis.

Conflict of interest: the authors report no conflicts of interest.

Contributions: all the authors contributed equally.

Received for publication: 7 April 2011.

Accepted for publication: 8 August 2011.

This work is licensed under a Creative Commons Attribution NonCommercial 3.0 License (CC BYNC 3.0).

(C) Copyright E. Kanonidou et al., 2011

Licensee PAGEPress, Italy

Eye Reports 2011; 1:e6

doi:10.4081/eye.2011.e6

tension and diabetes, in 1 patient, the 2 were smoking and minor local trauma, in 1 patient, the 2 were smoking and medication related to bleeding diathesis, and in 1 patient, the 2 were severe coughing and heavy exercise). In 6 of the patients, there were 3 risk factors (in 1 of the patients, the 3 were hypertension, medication related to bleeding diathesis, and lift of weight, in 1 patient, the 3 were hypertension, severe coughing and heavy exercise, in 1 patient, the 3 were hypertension, heavy exercise and minor local trauma, in patient, the 3 were hypertension, straining at stool and heavy exercise, in 1 patient, the 3 were hypertension, heavy exercise and medication related to bleeding diathesis, and in 1 patient, the 3 were heavy exercise, lift of weight and minor local trauma). In one patient, there were 4 risk factors: hypertension, severe coughing, heavy exercise and medication related to bleeding diathesis. In another one patient, there were 5 risk factors: hypertension, diabetes, straining at stool, medication related to bleeding diathesis and lift of weight.

The values of the blood coagulation parameters were between the normal limits [PLT: 256,866 (SD 51,709), PT: 11.38 (SD 0.85), aPTT: 26.02 (SD 2.45), INR:1.02 (0.63)]. It was interesting to observe that 29 patients (83\%) had elevated blood pressure during the ophthalmological examination (mean systolic value: $170 \mathrm{mmHg} \pm 15 \mathrm{mmHg}$ ) while 30 patients $(87 \%)$ mentioned that they considered that their condition should be medically treated despite the doctor's reassurance that $\mathrm{SCH}$ is a self-limiting condition. It is also worthy to mention that 2 patients $(6 \%)$ visited again the outpatients' department in a week's 
time, even though the SCH was partially absorbed, as they were still anxious regarding their condition.

\section{Discussion}

Though it is a non-vision threatening condition in most of the cases, SCH may reflect blood coagulation or underlying systemic disorders. A study by Pitts, et al. confirms that blood pressure is higher in SCH than in a control group. According to this study, there is also a high incidence of hypertension in patients with SCH referring themselves to the ophthalmologists, which persists on subsequent assessment. This finding was found true even when the patient attributed the SCH to eye rubbing or to a straining manoeuvre and whether or not the fundus shows early hypertensive changes. ${ }^{5}$ The high incidence of hypertension by established criteria suggests that hypertension may be an important aetiological factor in $\mathrm{SCH}$ and it is recommended that all patients with this condition have their blood pressure checked and be referred to the general practitioner. ${ }^{5}$ A further study by Mimura, et al. suggested that the cause of SCH in hypertensive patients is microvascular damage, which is more common in hypertensive patients than in otherwise healthy patients. ${ }^{2}$ In our investigation, it was interesting to observe that blood pressure was elevated in the vast majority of the patients with spontaneous ipsilateral SCH, a fact that might possibly be related to their concerns about their condition. $\mathrm{SCH}$ develops in patients with local trauma, or a trauma associated with retrobulbar hemorrhage or ruptured globe. ${ }^{6} \mathrm{SCH}$ is also recognized in whooping cough, sneezing, constipation or other forms of straining (Valsalva) and even strangulation, where the mechanism is thought to be raised venous pressure..$^{2,3,6}$ Recurrent, bilateral and severe subconjunctival hemorrhages mandate the search for an underlying etiology, such as a blood dyscrasia, blood clotting disorder, or recurrent increased intrathoracic pressure caused by repetitive vomiting or coughing spells. ${ }^{7}$ These risk factors were also assessed in our investigation. It has also been reported that SCH can be a feature of diabetes. ${ }^{8}$ Patients with a bleeding disorder, such as haemophilia, or others under anti-platelet or anticoagulant medication may be more prone to having a SCH. In our study, the coagulation blood tests were normal in all the patients. SCH presents more commonly as a spontaneous event without these etiological factors, especially in the elderly or arteriosclerotic patients. ${ }^{9} \mathrm{~A}$ recent study by Mimura, et al . reported that the peak age of occurrence of SCH was between 61 and 70 years. Fourteen

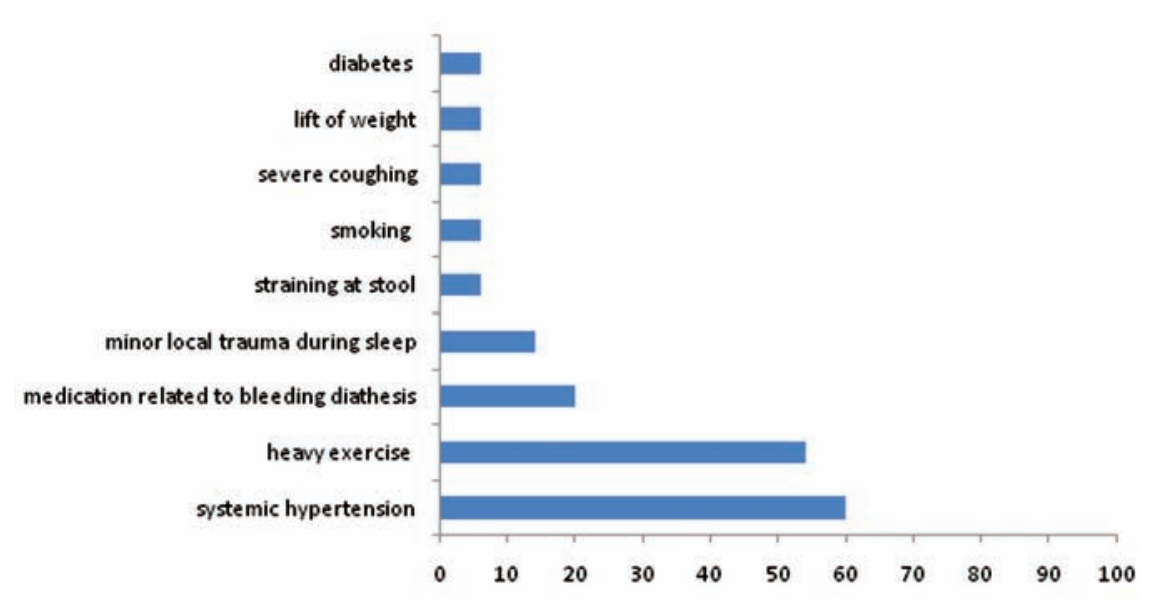

Figure 1. Risk factors related to spontaneous ipsilateral subconjunctival hemorrhage in the participants in our investigation (\% percentage).

patients (77.7\%) in that study had trauma or contact-lens-induced injury, and 4 patients (22.3\%) among the younger patients aged 0-40 years had an unknown etiology. Among the older patients aged 61-94 years, the chief risk factor for SCH was hypertension (47.5\%), followed by unknown etiology (39.4\%) and then diabetes (13.1\%). ${ }^{2} \mathrm{SCH}$ is self-limiting and typically resolves in two to three weeks, without any treatment required. Artificial teardrops can be given if a mild ocular irritation related to corneal dryness or dellen formation from an elevation of conjunctiva adjacent to the cornea is present. Some authors recommend further investigation of patients having $\mathrm{SCH}$ while others suggest that reassurance alone is required. ${ }^{10}$ In our investigation it was interesting to observe the high percentage of patients requesting prescribed medication for the treatment of their condition as well as the fact that a proportion of patients visited again the outpatient department for further consultation despite the doctor's reassurance at their initial visit. The reason for the patients' anxiety can be contributed to the fact that $\mathrm{SCH}$ can be a source of worry and an embarrassment to the patients, mainly due to its impressive appearance, but also to the mistaken belief that SCH is related to elevated intraocular pressure, strokes, or other serious circulatory system disorders. Our study provides documentation regarding the potential risk factors associated with $\mathrm{SCH}$. It is interesting to observe the high incidence of hypertension among the patients with SCH. It is possible that the high incidence of hypertension is related to patients' concerns about their condition. Nevertheless, it is highly recommended that the blood pressure be checked in all patients with SCH at the ophthalmic exam visit and that the patients should be referred to a general practitioner for further management if indicated.

\section{References}

1. Kanski JJ. Clinical Ophthalmology. A systematic approach. 2nd ed. Boston, Massachusetts: Butterworth-Heinemann; 2007. p. 629.

2. Mimura T, Usui T, Yamagami S, et al. Recent causes of subconjunctival hemorrhage. Ophthalmologica 2010;224:133-7.

3. Kaimbo Wa Kaimbo D. Epidemiology of traumatic and spontaneous subconjunctival haemorrhages in Congo. Bull Soc Belge Ophtalmol 2009;311:31-6.

4. Leiker LL, Mehta BH, Pruchnicki MC, Rodis JL. Risk factors and complications of subconjunctival hemorrhages in patients taking warfarin. Optometry 2009; 80:227-31.

5. Pitts JF, Jardine AG, Murray SB, Barker NH. Spontaneous subconjunctival haemorrhage-a sign of hypertension? Br J Ophthalmol 1992;76:297-9.

6. op de Coul ME, Budde JH. Diagnostic image (136). A boy with coughing fits and subconjunctival hemorrhage. Subconjunctival hemorrhage secondary to whooping cough. Ned Tijdschr Geneeskd 2003;147:805.

7. Trevor-Roper PD, Curran PV. The eye and its disorders. 2nd ed. 0xford: Blackwell Scientific; 1984. pp. 341-367.

8. Fukuyama J, Hayasaka S, Yamada K, Setogawa T. Causes of subconjunctival hemorrhage.0phthalmologica 1990;200:637.

9. Groomer AE, Terry JE, Westblom TU. Subconjunctival and external hemorrhage secondary to oral anticoagulation. J Am Optom Assoc 1990;61:770-5.

10. Alder FH. Gifford's textbook of ophthalmology. 4th ed. Philadelphia: Saunders; 1949. pp. 196-219. 